\title{
Survey on Recent Research and Implementation of Ant Colony Optimization in Various Engineering Applications
}

\author{
Chandra Mohan B \\ Department of computer science \& Engineering, \\ Anna University \\ Chennai, India \\ abc.phd@hotmail.com, +91-9976611188 \\ Baskaran R \\ Asst. Prof., Department of computer science \& Engineering, \\ Anna University \\ Chennai, India \\ baaski@cs.annauniv.edu, 9094022331 \\ Received 7 September 2010 \\ Accepted 7 February 2011
}

\begin{abstract}
Ant colony optimization (ACO) takes inspiration from the foraging behaviour of real ant species. This ACO exploits a similar mechanism for solving optimization problems for the various engineering field of study. Many successful implementations using ACO are now available in many applications. This paper reviewing varies systematic approach on recent research and implementation of ACO. Finally it presents the experimental result of ACO which is applied for routing problem and compared with existing algorithms.
\end{abstract}

Keywords: Swarm Intelligence, Ant colony optimization, Soft-computing, Engineering Applications

\section{Introduction}

Swarm intelligence is a new discipline of study that contains relatively optimal approach for problem solving which is the imitations inspired from the social behaviours of insects and of other animals, for ex: Ant colony optimization algorithm, artificial honey bee algorithms. The field of "Ant Algorithm" studies models derived from the observation of real ants' behaviour, and uses these models as a source of inspiration for the design of novel algorithms for the solution of optimization and distributed control problems. The main idea is that the self-organizing principles which allow the highly co-ordinated behaviour of real ants can be exploited to co-ordinated populations of artificial agents that collaborate to solve computational problems. Several different aspects of the behaviour of ant colonies have inspired different kinds of ant algorithms, for ex: foraging, division of labour, brood sorting, and co-operative transport. One of the most successful examples of ant algorithms is known as "Ant Colony Optimization (ACO)". ACO is inspired by the foraging behaviour of ant colonies, and targets the discrete optimization problems.

Pierre-Paul Grasse [1] observed that some species of termites react to what he called "significant stimuli". This French Entomologist observed that the effects of these reactions can act as new significant stimuli for both the insect that produced them and for the other insects in the colony. Grasse used the term stigmergy to describe this particular type of communication in which the "workers are stimulated by the performance they have achieved". Biologists have shown that it is often sufficient to consider stigmergic, indirect 


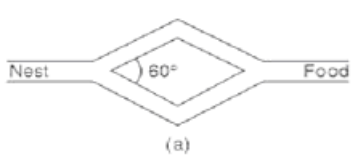

(a) Link with equal length

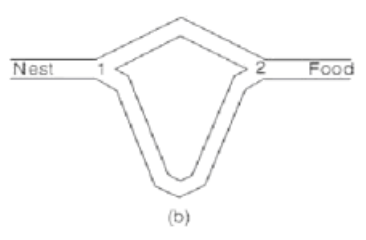

(b) Link with different lengt
Fig 1: Experimental setup for the double bridge experiment

communication to explain how social insects can achieve self-organization. Stigmergy is an indirect, nonsymbolic form of communication mediated by the environment and the stigmergic information is local, it can only be accessed by those insects that visit the locus in which it was released or its immediate neighbourhood.

In many ant species, ants walking to and from a food source deposit on the ground a substance called pheromone. Other ants perceive the presence of this pheromone and tend to follow the paths where pheromone concentration is higher. Through this mechanism, ants are able to identify and transport food to their nest in a remarkably effective and easy way. Deneubourg et al. [2] thoroughly investigated the pheromone laying and following behaviour of ants. In an experiment known as the "double bridge experiment", the nest of a colony of Argentine ants was connected to a food source by two bridges of equal lengths. The author used the term Argentine ants for the ants which identifies the path, simply says the predictor of the path. Refer Figure 1(a) \& (b) for the way of ACO decision making. The argentine ants always spread the work place and searching other possible routes. In such a setting, ants start to explore the surroundings of the nest and eventually reach the food source. Along their path between food source and nest, Argentine ants deposit pheromone. Initially, each ant randomly chooses one of the two bridges. However, due to random fluctuations, after some time one of the two bridges presents a higher concentration of pheromone than the other and, therefore, attracts more ants. This brings a further amount of pheromone on that bridge making it more attractive with the result that after some time the whole colony converges toward the use of the same bridge. This colony-level behaviour, based on

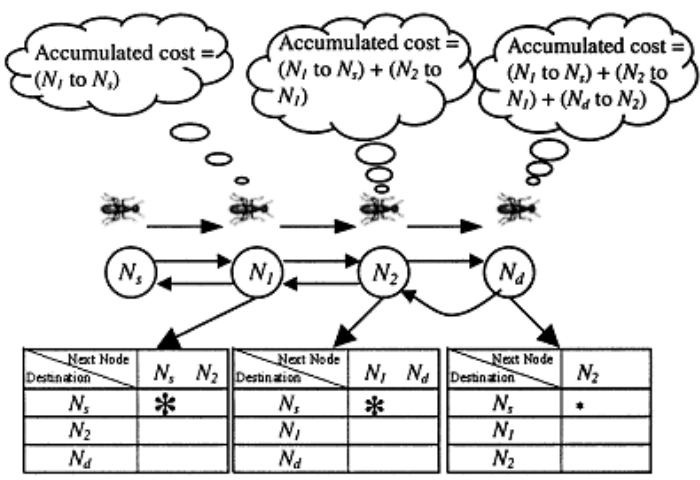

a) Pheromone update policy of Subramanian et al [52]

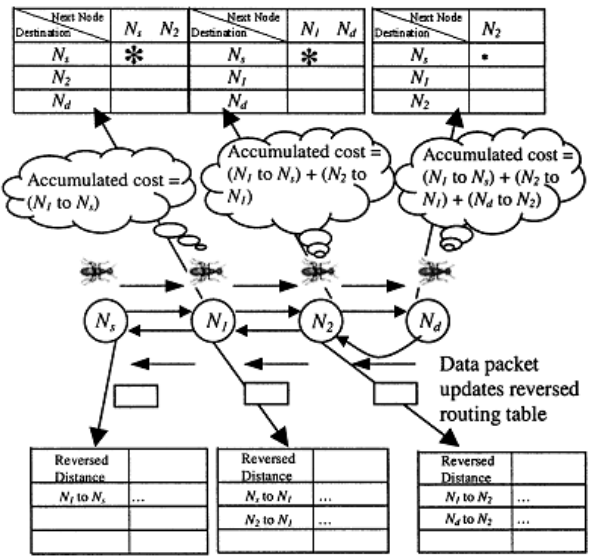

b) Pheromone update policy of Heusse et al..[53]

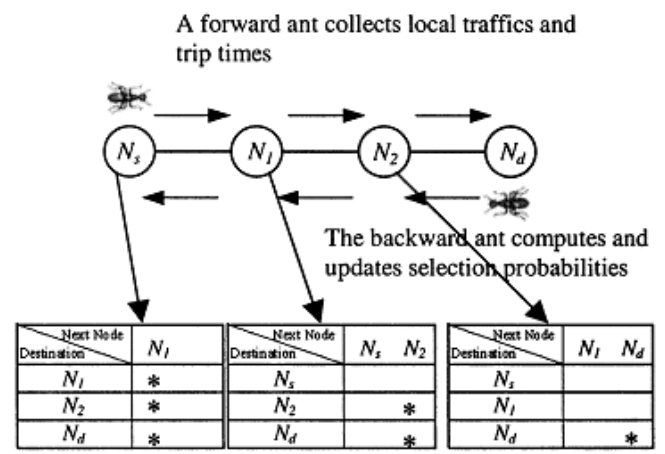

c) Pheromone update policy of Caro and Dorigo.[54]

Fig 2 Various pheromone update policy autocatalysis, that is, on the exploitation of positive feedback, can be used by ants to find the shortest path between a food source and their nest.

Goss et al. [3] considered a variant of the double bridge experiment in which one bridge is significantly 
longer than the other; refer the double bridge, Figure 1(b). In this case, the stochastic fluctuations in the initial choice of a bridge are much reduced and a second mechanism plays an important role: the ants choosing by chance the short bridge are the first to reach the nest. The short bridge receives, therefore, pheromone earlier than the long one and this fact increases the probability that further ants select it rather than the long one.

Pasteels et al. [4] developed a model of the observed behaviour: assuming that at a given moment of time, $\mathrm{m} 1$ ants have used the first bridge and $\mathrm{m} 2$ ants have used the second one, the probability $\mathrm{p} 1$ for an ant to choose the first bridge is:

$$
p_{1}=\frac{\left(m_{1}+k\right)^{h}}{\left(m_{1}+k\right)^{h}+\left(m_{2}+k\right)^{h}},
$$

Where parameters $\mathrm{k}$ and $\mathrm{h}$ are constant and which is to be fitted to the experimental data-obviously, and p2 $=1-\mathrm{p} 1$.

Suppose that there are four ants $\left(\mathrm{A}_{1}, \mathrm{~A}_{2}, \mathrm{~A}_{3}\right.$, and $\left.\mathrm{A}_{4}\right)$ and two routes ( $R 1$ and $R 2$ ) leading to a food source $\left(F_{0}\right)$, where $R_{1}$ and $R_{2}$ such that $R_{1}>R_{2}$ and $R_{1}=2 * R_{2}$. Initially, all ants are at the decision point $\mathrm{N}_{\mathrm{e}}$ and they have to select between $R_{1}$ and $R_{2}$ to reach $F_{o}$.

1) At $\mathrm{N}_{\mathrm{e}}$, all ants have no knowledge about the location of food $\left(\mathrm{F}_{0}\right)$. Hence, they randomly select from $\left\{R 1, R_{2}\right\}$. Suppose that $A_{1}$ and $A_{2}$ choose $R_{1}$, and $A_{3}$ and $\mathrm{A}_{4}$ choose $\mathrm{R}_{2}$.

2) As $A_{1}$ and $A_{2}$ move along $R_{1}$, and $A_{3}$ and $A_{4}$ move along $R_{2}$, they leave a certain amount of pheromone along their paths $\tau_{\mathrm{R} 1}$ and $\tau_{\mathrm{R} 2}$, respectively.

3) Since $R_{1}>R_{2}, A_{3}$ and $A_{4}$ reach $F_{0}$ before $A_{1}$ and $A_{2}$. When $A_{3}$ and $A_{4}$ pass $R_{2}$ to reach $F_{0}, \tau_{R 2}=2$, but $A_{1}$ and $A_{2}$ have yet to reach $F_{0}$ and $\tau_{R 1}=0$. To return to $N_{e}$ from $F_{0}, A_{3}$ and $A_{4}$ have to choose between $R_{1}$ and $R_{2}$. At $F_{0}, A_{3}$ and $A_{4}$ detect that $\tau_{R 2}>\tau_{R 1}$, hence they are more likely to select $R_{2}$.

4) As $A_{3}$ and $A_{4}$ pass $R_{2}$ for the second time to reach $N_{e}, \tau_{R 2}$ is incremented to 4 . The increase in $\tau_{R 2}$ further consolidates $\mathrm{R}_{2}$ as the shorter path. When $\mathrm{A}_{1}$ and
$\mathrm{A}_{2}$ reach $\mathrm{F}_{0}, \tau_{\mathrm{R} 2}=4$ and $\tau_{\mathrm{R} 1}=2$. Hence, $\mathrm{A}_{1}$ and $\mathrm{A}_{2}$ are more likely to select $\mathrm{R}_{2}$ to return to $\mathrm{N}_{\mathrm{e}}$.

In this example, any ant at $\mathrm{F}_{0}$ (respectively, $\mathrm{N}_{\mathrm{e}}$ ) will be able to determine the optimal path once $A_{3}$ and $\mathrm{A}_{4}$ reach $\mathrm{F}_{0}$. If an ant is at a choice point when there is no pheromone (e.g., initially at $\mathrm{N}_{\mathrm{e}}$ ), it makes a random decision with a probability of 0.5 of choosing $R_{1}$ or $R_{2}$. However, when pheromone is present (e.g., when the ant is at $\mathrm{F}_{0}$ ), there is a higher probability that it will choose the path with the higher concentration of pheromone.

Furthermore, there are some ACO approaches that adopt privileged pheromone lying in which ants only deposit pheromones during their return trips, the various pheromone update policy is shown in fig 2. In addition, most ACO approaches are inspired by the problem solving paradigms of ants rather than building exact replicas of biological ants. In using artificial ants for problem solving, some of the features and capabilities of bio-logical ants (e.g., using visual and marks) may be omitted, and other additional techniques (e.g., heuristic functions) may be used to complement and supplement the use of pheromone.

Dorigo et al. proposed Ant System (AS) [5] and Elitist AS [6] [7] is the significant implementation for engineering applications. Dorigo contributed several research works in the swarm intelligence [8], simply says Dorigo may designate as the father of ACO. Ant-Q developed by Gambardella \& Dorigo [9], Ant Colony System developed by Dorigo \& Gambardella [10], MaxMin AS developed by Stutzle \& Hoos [11], Rank-Based AS developed by Bullnheimer et al. [12], ANTS proposed by Maniezzo [13], BWAS proposed by Cordon et al. [14], Hyper-Cube AS proposed by Blum et al. [15] are some of the successful ant optimization algorithm.

\section{Recent research in $\mathrm{ACO}$}

This paper reviews the recent systematic approach of ACO on various engineering field of application, papers published only in 2010 are considered for in this survey.

\subsection{Scheduling:}

Job Scheduling problems have a vital role in recent years due to the growing consumer demand for variety, reduced product life cycles, changing markets with global competition and rapid development of new 
technologies. The Job Shop Scheduling Problem (JSSP) is one of the most popular scheduling models existing in practice, which is among the hardest combinatorial optimization problems. The instance definition of job scheduling problem is as follows:

- A number of independent (user/application) jobs to be scheduled.

- A number of heterogeneous machines candidates to participate in the planning.

- The workload of each job (in millions of instructions).

- The computing capacity of each machine (in mips).

- Ready time indicates when machine $\mathrm{m}$ will have finished the previously assigned jobs.

- The Expected Time to Compute (ETC) matrix ('nb' jobs $\times$ ' $n b$ ' machines) in which ETC[i][j] is the expected execution time of job ' $\mathrm{i}$ ' in machine ' $\mathrm{j}$ '.

Many approaches, such as, Simulated Annealing (SA), Tabu Search (TS), Genetic Algorithm (GA), Ant Colony Optimization (ACO), Neural Network (NN), Evolutionary Algorithm (EA) and other heuristic approach have been successfully applied to JSSP. For improving the performance of EA, several researches integrated some optimization strategies into the EA [16]. Also, the study of interaction between evolution and learning for solving optimization problems has been attracting much attention. The diversity of these approaches has motivated Li-Ning Xing et al [17]. The authors proposed a frame work called KnowledgeBased Heuristic Searching Architecture (KBHSA), which integrates knowledge model and heuristic searching model to search an optimal solution. The performance of this architecture in the instantiation of the Knowledge-Based Ant Colony Optimization (KBACO) which is applied to common benchmark problems. Experimental results show that KBACO algorithm outperforms previous approaches for solving the FJSSP.

Wang Chen et al [18] proposed an efficient hybrid algorithm for resource-constrained project scheduling. This hybrid algorithm is known as the ACOSS algorithm which combines Scatter Search (SS) with ACO. Research on ACO has shown that improved performance can be obtained by stronger exploitation of the best solutions found during the search [19] [20]. Yet, using a greedier search potentially aggravates the problem of premature stagnation of the search. Therefore, the key to obtaining best performance of ACO algorithms is to combine an improved exploitation of the best solutions with an effective mechanism for avoiding early search stagnation. Combining exploitation of the best solutions with a problemdependent local search algorithm, the authors presents the hybrid algorithm. In this paper, as a first step, all ants in the ACO search the solution space and generate activity lists to provide the initial population for the SS . Then, although the SS improves all the ants' solutions, only the best solution (thus far) is used to update the pheromone trails. Finally, ACO searches the solution space again using the new pheromone trails. In other words, the SS uses the previous population constructed by $\mathrm{ACO}$, which subsequently updates the pheromone trails using the best solution from the SS, and searches again. In addition, a local search strategy is employed to improve the quality of solutions generated by ACO, and also as the improvement method in the SS. In this scheme, ACO and SS alternatively and cooperatively search the solution space until the termination criterion is satisfied. In each generation, ACO only executes once to generate the initial population for the SS, which then executes one or more times to improve the solutions.

Despite the interdependent relationship between the production scheduling and the maintenance planning, the two activities are generally planned and executed separately in real manufacturing systems. For many years the relationship between production and maintenance has been considered as a conflict in management decision. This situation stills the same because of the lack of communication regarding the scheduling requirements of each function. The conflicts may result in an unsatisfied demand or machine breakdowns if the production and maintenance services do not respect the requirements of each other. Several studies have been devoted to optimize the two functions, scheduling and maintenance, but separately. The majority of studies in scheduling taking into account maintenance adopt the approach called "machine scheduling with availability constraints" where the time interval of preventive maintenance actions as well as their number are known and fixed in advance. Berrichi et al [21] proposed a Bi-Objective ACO approach to optimize production and maintenance scheduling. This paper presents an algorithm based on ACO paradigm to solve the joint production and maintenance scheduling problem. This approach is developed to deal with the parallel machine case. This model is formulated according to a bi-objective 
approach to find trade-off solutions between both objectives of production and maintenance. Reliability models are used to take into account the maintenance aspect. To improve the quality of solutions found in our previous study, an algorithm based on Multi-Objective Ant Colony Optimization (MOACO) approach is developed. The goal is to simultaneously determine the best assignment of production tasks to machines as well as preventive maintenance periods of the production system, satisfying at best both objectives of production and maintenance.

Wei-Neng Chen et al [22] applied ACO in the cash flow monitoring and control in the project scheduling. Cash flow means the amount of cash being received and spent during a defined period of time. Without positive cash flows, basic obligations such as payments to suppliers and payrolls cannot be met. In project level, even a high-profit project may turn out to be a failure if cash short-fall suddenly occurs. As such, cash flow management in project level has attracted a considerable amount of research effort in recent years. A promising progress is to integrate cash flow management with the resource-constrained project-scheduling problem (RCPSP). The classical RCPSP is a problem of finding an optimal schedule that satisfies the resource and precedence constraints and minimizes the make-span (duration) of a project. Applications of the RCPSP can be found in a broad area of industrial projects, such as house building and software development. The author proposed a multimode RCPSP with discounted cash flows using ACO. For further studies, please refer the comprehensive survey of Herroelen et al. [23], Brucker et al. [24] and recent research in RCPSP [25][26]. The application of ACO requires setting up a construction graph and designing the pheromones and heuristic information. Based on the construction graph, the serial schedule generation scheme is applied for artificial ants to build solutions. In the process of this algorithm, each ant maintains a schedule generator and builds its solution following the rules of ACS using pheromones and heuristic information.

For schools, parks, and recreational areas, the maintenance of green areas is important, but it places a heavy burden on both manpower and budget. Hsin-Yun Lee et al [27] proposed a model to search for the minimum gardener manpower requirements and a nearoptimal maintenance schedule for the green areas. Unlike other applications of ACO, they grouped the ants into teams to represent gardeners and considered the path of an ant team a schedule solution. The authors implemented the proposed model using a decision support system called the Garden-Ant. In addition, the feasibility of the model was evaluated on a campus. The results of the evaluation indicated that the proposed model could provide an appropriate maintenance plan, including manpower estimation and an all-inclusive maintenance schedule. Because the complicated calculations are performed by the model instead of by an administrator, the maintenance planning of green areas can be accomplished in an easy and efficient manner.

\subsection{Traveling salesman problem:}

Let $\mathrm{V}=\{\mathrm{a}, \ldots, \mathrm{z}\}$ be a set of cities, $\mathrm{A}=\{(\mathrm{r}, \mathrm{s}): \mathrm{r}, \mathrm{s} € \mathrm{~V}\}$ be the edge set, and $\delta(r, s)=\delta(s, r)$ be a cost measure associated with edge $(\mathrm{r}, \mathrm{s}) \in \mathrm{A}$. The TSP is the problem of finding a minimal cost closed tour that visits each city once. In the case cities $\mathrm{r} \in \mathrm{V}$ are given by their coordinates $\left(\mathrm{x}_{\mathrm{r}}, \mathrm{y}_{\mathrm{r}}\right)$ and $\delta(\mathrm{r}, \mathrm{s})$ is the Euclidean distance between $r$ and $s$, then it is an Euclidean TSP. If $\delta(r, s) \neq$ $\delta(\mathrm{s}, \mathrm{r})$ for at least some $(\mathrm{r}, \mathrm{s})$ then the TSP becomes an Asymmetric TSP (ATSP).

The traveling salesman problem with time windows is a difficult optimization problem that arises, for example, in logistics. Manuel and Blumb [28] proposed a Beam-ACO for the traveling salesman problem with time windows. This paper deals with the minimization of the travel-cost. For solving this problem, this paper proposes a Beam-ACO algorithm, which is a hybrid method combining ACO with beam search. In general, Beam-ACO algorithms heavily rely on accurate and computationally inexpensive bounding information for differentiating between partial solutions. This work uses stochastic sampling as a useful alternative. An extensive experimental evaluation on seven benchmark sets from the literature shows that the proposed Beam-ACO algorithm is currently a state-of-the-art technique for the traveling salesman problem with time windows when travel-cost optimization is concerned.

Using ACO algorithms to obtain good solutions for combinatorial optimization problems has become very popular in recent years. In contrast to other kinds of randomized search heuristics such as Simulated Annealing for evolutionary algorithms, ACO algorithms have the ability to integrate knowledge about the problem into the construction of a new solution. In the 
case of a new combinatorial optimization problem, there is often some knowledge about the problem which can be incorporated into this kind of randomized search heuristic. Therefore, the main application of ACO algorithms lies in the field of combinatorial optimization and the first problem to which this kind of heuristic has been applied was the traveling salesperson problem. ACO is inspired by a colony of ants that search for a common source of food. It has been observed that ants are able to find a shortest path to such a source under certain circumstances by indirect communication. This communication is done by socalled pheromone values. The behavior of ants is put into an algorithmic framework to obtain solutions for a given problem. Solutions are constructed by random walks of artificial ants on a so-called construction graph, which has weights - the pheromone values - on the edges. Larger pheromone values lead to higher probability of the edges being traversed in the next walk. In addition, the random walk is usually influenced by heuristic information about the problem.

Frank Neumanna, and CarstenWitt [29], proposed an ACO for the minimum spanning tree problem. The authors presented the first comprehensive rigorous analysis of a simple ACO algorithm for a combinatorial optimization problem. In their investigations, they consider the minimum spanning tree (MST) problem and examine the effect of two construction graphs with respect to the runtime behavior. The choice of the construction graph in an ACO algorithm seems to be crucial for the success of such an algorithm. First, the input graph itself as the construction graph and analyze the use of a construction procedure that is similar to Broder's algorithm (Perform a random walk until the cover time) for choosing a spanning tree uniformly at random. After that, a more incremental construction procedure is analyzed. It turns out that this procedure is superior to the Broder's-based algorithm and produces additionally in a constant number of iterations an MST, if the influence of the heuristic information is large enough.

Xiao-ming You et al [30] proposed Parallel Ant Colony Optimization Algorithm (PQACO) based on Quantum dynamic mechanism for traveling salesman problem. In PQACO, the author explains the Parallel Evolutionary Algorithms (PEA) can be classified into three different models: Master-slaves PEA, Fine-grained PEA, Coarse-grained PEA and used this model for the travelling salesman problem of quantum computing applications.

\subsection{Clustering:}

Clustering, so-called set partitioning, is a basic and widely applied methodology. Application fields include statistics, mathematical programming (such as location selecting, graph theory, scheduling and assignment problems, etc.) and computer science (including pattern recognition, network partitioning, routing, learning theory, image processing and computer graphics, etc.). Clustering is mainly to group all objects into several mutually exclusive clusters in order to achieve the maximum or minimum of an objective function. Clustering is rapidly becoming computationally intractable as problem scale increases, because of the combinatorial character of the method. It is proved that, for specific object functions, clustering becomes an NPhard problem when the number of clusters exceeds 3 . Data clustering, which is an NP-complete problem of finding groups in heterogeneous data by minimizing some measure of dissimilarity, is one of the fundamental tools in data mining. Cluster analysis is one of the attractive data mining techniques that are used in many fields. One popular class of data clustering algorithms is the center based clustering algorithm. There are many methods applied in clustering analysis, like hierarchical clustering, partition-based clustering, density-based clustering, and artificial intelligencebased clustering. K-means is used as a popular clustering method due to its simplicity and high speed in clustering large datasets. However, K-means has two shortcomings: dependency on the initial state and convergence to local optima and global solutions of large problems cannot found with reasonable amount of computation effort. In order to overcome local optima problem lots of studies have been done in clustering. Many methods for local optimization are based on the notion of a direction of a local descent at a given point. A local improvement of a point in hand can be made using this direction. As a rule, modern methods for global optimization do not use directions of global descent for global improvement of the point in hand. From this point of view, global optimization algorithm based on a dynamical systems approach (GOP) is an unusual method. Its structure is similar to that used in local optimization: a new iteration can be obtained as an improvement of the previous one along a certain 
direction. In contrast with local methods, is a direction of a global descent and for more diversification combined with Tabu search.

Ali Maroosi and Babak Amiri [31] proposed a Hybrid Global Optimization (HGOP) based on a dynamical systems approach algorithm. In this paper, the author proposed the application of hybrid global optimization algorithm based on a dynamical systems approach and compared the propose HGOP with other algorithms in clustering, such as GAK, SA, TS, and $\mathrm{ACO}$, by implementing them on several simulation and real datasets.

\subsection{Electrical Engineering:}

Economic dispatch(ED) problem is one of the mathematical optimization issues in power system operation and it attracts researchers' attention all the way. With an increasing concern over the environmental pollution caused by thermal power plants, environmental/economic dispatch (EED) problem has drawn much more attention for a good dispatch scheme from it would not only result in great economical benefit, but also reduce the pollutants emission. Different techniques have been reported in the literature pertaining to EED problem, including conventional approaches such as weighted mini-max method, direct analytical solution method, linear programming and 1constraint method, and artificial intelligence technology such as genetic algorithm, particle swarm optimization, fuzzy set theory and evolutionary programming. In principle, these approaches usually employed to deal with EED problems can be classified into two categories, namely, Lagrange multiplier methods and a multi-objective stochastic search technique. Many researchers have performed studies in this field. tradeoff relations between cost and emission is the main objective of this problem. Jiejin Cai et al [32] proposed a multi-objective chaotic ant swarm optimization for EED. This paper developed a multi-objective chaotic ant swarm optimization (MOCASO) method for solving the EED problems of thermal generators in power systems considering both economic and environmental issues. In MOCASO method, Pareto-dominance is employed to handle multi-objective problems, and fuzzifying, fitness sharing and turbulence factor perturbing techniques are also embedded. The proposed method was successfully employed to solve the EED problems in three test systems considering some constraints, such as power balance constraints and generation limits constraints. The author produced numerical simulation results which indicated that the MOCASO method is feasible and effective for solving EED problem for power systems.

Saravuth Pothiya et al [33] proposed an Ant colony optimisation for economic dispatch problem with nonsmooth cost functions. This paper presents a novel and efficient optimisation approach based on the ACO for solving the economic dispatch (ED) problem with nonsmooth cost functions. In order to improve the performance of ACO algorithm, three additional techniques, i.e. priority list, variable reduction, and zoom feature are presented. To show its efficiency and effectiveness, the proposed ACO is applied to two types of ED problems with non-smooth cost functions. First, the ED problem with valve-point loading effects consists of 13 and 40 generating units. Second, the ED problem considering the multiple fuels consists of 10 units. Additionally, the results of the proposed ACO are compared with those of the conventional heuristic approaches. The experimental results of this paper show that the proposed ACO approach is comparatively capable of obtaining higher quality solution and faster computational time.

Reactive power management is essential to transfer real energy and support power system security. Developing an accurate and feasible method for reactive power pricing is important in the electricity market. In conventional optimal power flow models the production cost of reactive power was ignored. Abbas Ketabi et al [34] proposed an Application of the ant colony search algorithm to reactive power pricing in an open electricity market. In this paper, the production cost of reactive power and investment cost of capacitor banks were included into the objective function of the OPF problem. Then, using ant colony search algorithm, the optimal problem was solved. Marginal price theory was used for calculation of the cost of active and reactive power at each bus in competitive electric markets. The application of the proposed method on IEEE 14-bus system is confirms its validity and effectiveness. This ACO algorithm has the following features: 1) The points in feasible region are regard as "ants". After some iteration, the ants will centralize at the optimum points which could be one or more points. There are two choices for an ant in the each iteration: moving to other ants' point or searching in neighborhood. 2) The 
iteration would be guided by changing the distribution of intensity of pheromone in feasible region. 3) Sequential quadratic programming (SQP) is used as neighborhood-searching algorithm to improve the precision of convergence. The roulette wheel selection and disturbance are used to prevent the sub-optimization in ACO. The result of this paper shows that the effects of various factors on reactive power price on several case studies.

Fuel cell power plants (FCPPs) have been taken into a great deal of consideration in recent years. The continuing growth of the power demand together with environmental constraints is increasing interest to use FCPPs in power system. Since FCPPs are usually connected to distribution network, the effect of FCPPs on distribution network is more than other sections of power system. One of the most important issues in distribution networks is optimal operation management (OOM) which can be affected by FCPPs. Taher Niknam et al [35] proposed a practical algorithm for optimal operation management of distribution network including fuel cell power plants. In this paper, the author proposes a new approach for optimal operation management of distribution networks including FCCPs. In the article, they consider the total electrical energy losses, the total electrical energy cost and the total emission as the objective functions which should be minimized. Whereas the optimal operation in distribution networks has a nonlinear mixed integer optimization problem, the optimal solution could be obtained through an evolutionary method. The authors uses a new evolutionary algorithm based on Fuzzy Adaptive Particle Swarm Optimization (FAPSO) to solve the optimal operation problem and compared this method with Genetic Algorithm (GA), Particle Swarm Optimization (PSO), Differential Evolution (DE), ACO and Tabu Search (TS) over two distribution test feeders.

\subsection{Routing Algorithm:}

The comparison of traditional RIP routing versus ACO routing algorithms is shown in Fig 5. Sudip Misra et al [36] proposed a low-overhead Fault-Tolerant Routing (FTAR) algorithm for Mobile Ad Hoc Networks (MANET). MANETs are self-organizing and selfconfiguring networks having nodes connected by wireless links. They are infrastructure-less and nodes in them can join or leave at any point of time. There is no centralized control in MANETs, all nodes behave as routers for each other, and data packets are transferred for node to node in a multihop fashion. The mobile nodes, which are inherently resource constrained, exhibit various kinds of faulty behavior. Faulty behavior may be transient or permanent and may be due to hardware or software problems. In such a scenario, a faulty node may not forward packets. Absence of any underlying infrastructure makes it difficult to keep these devices monitored. Moreover, the adversarial

Fig 3 First Level functionality diagram of Ant colony based data collection 
environment in MANETs makes the situation worse. Making routing decisions oblivious of these nodes will significantly degrade the performance of any routing protocol and can also threaten its prime objectives. Fault-tolerant routing protocols address this problem by exploring the network redundancy through multipath routing techniques. FTAR, is essentially aimed at applying the ACO concepts on the set of paths obtained by the source routing algorithm. So, unlike in the traditional ACO-based routing schemes, the ants defined by FTAR are not route finding ants, but are ants that work on the paths already at the disposal of the source node. FTAR introduces the notion of worker ants which are special control packets sent out by the source node to determine the fault-tolerance of a particular path. The worker ants can be sent by the source node reactively or proactively depending on the load on the system. Reactive ants are sent during a data session at a predetermined rate. For example, $m$ worker ants can be sent after every ' $n$ ' data packets. Proactive ants can be sent by the source node when the system is idle and this helps decision making for a data session in the future. Unlike the ants in the previously mentioned traditional ACO-based routing algorithms, worker ants work on paths and not on individual links - they are sent out by the source node and they retrace their path from the destination back to the source. They deposit pheromones on the paths when they reach the source back from the destination. The pheromone values are stored at the source node corresponding to each path. In case the nodes of a path are faulty or become faulty due to some activity, the algorithm decreases the confidence of such a path. These confidence values are used by the source node to make its decision regarding data routing. A high confidence value indicates a good fault-tolerant path implying a high packet delivery rate.

\subsection{Digital Image Processing:}

Image segmentation refers to the process in which spatially connected pixels that share certain visual characteristics are assigned with the same label. The major goal is to partition an input image into multiple segments so that objects and boundaries could be located and the image could become more meaningful and easier to analyze. In image processing and computer vision, segmentation has long been considered one of the most important problems since it plays an indispensable preliminary processing role for semantic analysis and many other advanced tasks. Indeed, accurately segmenting out objects or regions that appeal to the human vision is a significant issue. Though much emphasis has been put on this topic and many approaches have been proposed, it is still challenging to segment natural images due to their inherent complexity. The simplest case might be the segmentation of an image with distinct and homogeneous foreground objects. In this case, techniques such as threshold and edge detection using gradient information could be sufficient, even though they are relatively simple. Recent researches have also concentrated on applying threshold with intelligent algorithms like ACO and fuzzy measures so that more adaptive and accurate decisions could be made to achieve better results.

The iterative optimization of traditional Fuzzy CMean (FCM) clustering and segmentation algorithm is essentially a local searching method, which is likely to fall onto a local minimum point and is very sensitive to the initialization condition of cluster centers and centroid number. Usually, initialization is carried out based on certain experience. Clustering result depends largely on whether parameters have been properly chosen. When confronted with massive data of high dimensions, it is hard to both manually and properly set parameters without repetitive experiments, which is a laborious operation likely to generate sub-optimized image segmentation results. The author improved Ant System to initialize FCM in view of the drawbacks. The main underling principle is to use the robustness of Ant colony algorithm to overcome FCM's sensitiveness to the initialization condition. Moreover, its intelligent searching ability will help to further achieve optimization. In order to improve its performance for clustering tasks, relevant modifications should be made for AS. AS is well known to suffer from high computational complexity, as indicated in many previous researches. When applied in clustering problems, AS tends to be time consuming because for every pixel in an image, distances and pheromone concentration on the paths that lead to all the other ants have to be calculated, requiring tremendous computation. In addition, computation requirement will be tripled if every ant is a 3-D vector instead of a 1-D one. To solve this problem, Zhiding Yu et al [37] proposed an adaptive unsupervised approach toward pixel clustering and color image segmentation. The 
author chooses M0 cluster centers based on color quantization and hence every ant only needs to calculate its distances to these "food sources". Computation can be reduced because $\mathrm{M} 0$ is usually much smaller than $\mathrm{N}$, the total number of pixels. And further confine the computation by setting the cluster radius. If the distance between an ant and a cluster center is larger than a given radius, then its probability of going to that "food source"' is set to zero. In this fashion, ants will simply ignore clusters that are too far away. This may further bring higher clustering accuracy and smaller computational complexity to the algorithm. In each round of iteration, ants previously classified will no longer be considered. Only those unclassified will take part in the clustering process which is a hard probabilistic partition. The algorithm will accelerate as more and more ants become classified. There are two possible convergence conditions: either all ants are classified, or there are still unclassified ants but the clustering centroids remain unchanged for a certain number of iterations. Another concerned problem is stagnation, which comes from excessive pheromone accumulation on a single path. AS could easily get into stagnation as a result of its product form of heuristic information and pheromone concentration in the probability equation. This situation is especially likely to happen when solving clustering problem with AS, for massive number of ants may choose the same cluster center at a time, leaving intense pheromone concentration which attracts even more ants in the next round. The author propose a probability equation with the summation form of heuristic information and pheromone concentration in order to improve its robustness against massive build up of pheromone. When an ant is in close proximity to a food source, heuristic information will play the dominant role and the ant will most likely be attracted to this food source. This kind of formation helps to reduce the chance that an ant goes erroroneously to a food source much further away because of huge pheromone concentration at that point. They build a region adjacency graph for the clustered image. Eight-connection is applied to define spatial connectivity and spatially connected pixels in the same cluster are assigned with the same labels. They simply set a threshold for the minimum region pixel number to eliminate small regions and greedily merge two regions together according to their region dissimilarities. The stopping criterion would either be a threshold for the region dissimilarity, or a minimum region number. The dissimilarity measure could be elaborately designed to achieve better results, but for simplicity only the Euclidean distance between the two region mean feature vectors is considered in this paper. The author observed that, with these relatively simple operations, reasonable segmentations could be generated.

\subsection{Structural and Concrete Engineering}

The design of bridge piers is crucial for the design of pre-stressed concrete viaducts. The piers make up between $20 \%$ and $50 \%$ of the total cost of the viaduct depending on pier heights and foundation conditions. Rectangular hollow cross-sections as described in the present paper are most frequently used. Current designs of such reinforced concrete (RC) structures are highly conditioned by the experience of structural engineers. Design procedures usually adopt cross-section dimensions and material grades based on commonly sanctioned practice. Once the geometry and materials of the structure are specified, the reinforcement of the pier is tentatively defined according to experience. The firstorder stress resultants are analyzed and second-order (buckling) stress resultants are then estimated according to simplified and conservative formulae or following a more general method that accounts for second-order deformations and includes the non-linear stiffness of the column. Tentative passive reinforcement must then satisfy the limit states prescribed by concrete codes. Should the dimensions, the material grades or the reinforcement be insufficient, the structure is redefined on a trial-and-error basis. This process leads to safe designs, but the cost of the RC pier is, consequently, highly dependent upon the experience of the structural designer. In contrast to designs based on experience, artificial intelligence has been applied to a variety of fields including the solution of constrained problems. The design of RC structures is a problem of selecting design variables as subject to structural constraints for which artificial intelligence is aptly suited. Exact methods and heuristic methods are the two main approaches to structural optimization. Exact methods are usually based on the calculation of optimal solutions following iterative techniques of linear programming of the expressions of the objective function and the structural constraints [1, 2]. These methods are computationally quite efficient when the number of variables is limited since they require a small number of 
iterations. However, they must solve the problem of linear conditioned optimization in each iteration of the analysis, which is computationally laborious when there are a large number of variables. In addition, exact methods require explicit expressions for the constraints which are not available in the present case of a nonlinear buckling column. The second approach involves the heuristic methods based on artificial intelligence procedures. These methods include a wide range of artificial intelligence search algorithms, such as genetic algorithms, simulated annealing, threshold accepting, tabu search, ant colonies, and the like [3-7]. These methods involve simple algorithms, but they also require a considerable computational effort, since they include a large number of iterations in which the objective function is evaluated and the structural constraints are checked.

Francisco J. Martínez et al proposed the economic optimization of reinforced concrete (RC) bridge piers with hollow rectangular sections and describes the efficiency of three heuristic algorithms: two new variants of the ACO algorithm, the genetic algorithm (GA) and the threshold acceptance (TA) algorithm. The GA and TA are used for comparison with the new ACO algorithms. The total number of variables is 95. All variables are discrete in this analysis. The calibration of the new ACO algorithm recommended a 250-member ant population and 100 stages. The best solution costs 69,467 euros, which means savings of about $33 \%$ as compared to experience-based design.

\section{ACO Implementation Performance Evaluation}

The proposed system involves swarm intelligence. The swarm intelligence - Ant colony is used for routing in the optimal congestion free route.

Ant colony algorithms [11], [12] have been inspired by the behavior of the real ant colony. The algorithm can find the optimum solution by generating artificial ants. As the real ants search their environment for food, the artificial ants search the solution space. The probabilistic movement of ants in the system allows the ants to explore new paths and to re-explore the old visited paths. The strength of the pheromone deposit directs the artificial ants toward the best paths and the pheromone evaporation allows the system to forget old information and avoid quick convergence to suboptimal solutions. The probabilistic selection of the paths allows searching large number of solutions. ACO has been applied successfully to discrete optimization problems such as the traveling salesman problem [13], routing [14], and [15]. A number of proofs for the convergence to the optimum path of the ACO can be found in [16] and [17]. The implementation of proposed system [18] [19] in the wired environment which provides optimum result and suggested traffic free routing.

The ant agents move in the network randomly to scan large number of network nodes. While it is moving, it collects information about the network and delivers it to the network nodes. The algorithms of this category are not using the agents to optimize the paths as in S-ACO or S-ACO meta-heuristic [7]. It is just used to deliver more updated information about the network to the network nodes, which speeds up the optimization process. This category of ant like mobile agent algorithm is already successfully implemented in the

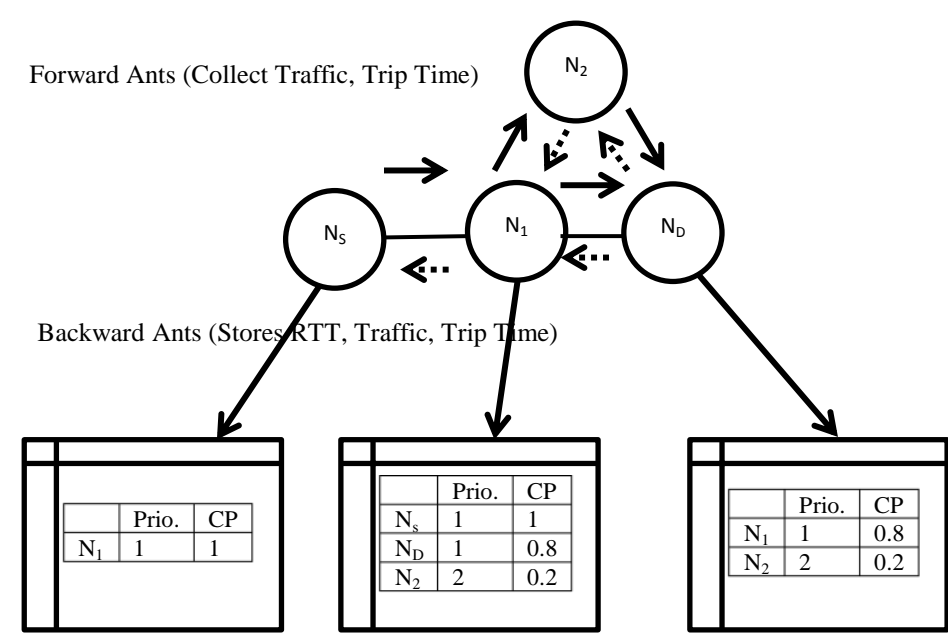

Fig 4: Flow Diagram of Ants movement and Functionality in the Proposed ACO

GPS / ant-like routing algorithm for mobile ad hoc networks and ant adhoc on-demand distance vector routing (AODV) hybrid routing protocol.

Every node in the network can function as a source node, destination node, and/or intermediate node. Every node has a pheromone table and a routing table. The routing table can be constructed based on the state 
transition rule and pheromone update policy. The following random proportional rule is applied as State transition rule: for destination $\mathrm{D}$, at node $\mathrm{i}$, the probability of selecting a neighbor $\mathrm{j}$ is

$$
\operatorname{prob}(D, i, j)=F u n(T D, i, j, \eta)----i f, j \in N
$$

Where TD is the pheromone value corresponding to neighbor $\mathrm{j}$ at node $\mathrm{i}$ and $0<\mathrm{TD}<1$ is the local heuristic value of the link $(i, j) /$ or node j. $0<\eta<1$. is the value can represent neighbor's information (i.e., neighbors queue delay, battery's remaining energy, processing power, link's signal-to-noise ratio, link's bandwidth, bit-error rate, etc.). Fun (TD, i, j, $\eta$ ) is a function in TD and $\eta$ (this function value is high when TD and $\eta$ are high). $\mathrm{N}$ is the set of all feasible neighbor nodes defined by the ant's information and the routing constraints (i.e., the guarantee of loop free). Assuming that at a given moment in time $\mathrm{m} 1$ ants have used the first bridge and $\mathrm{m} 2$ the second one, the probability $\mathrm{p} 1$ for an ant to choose the first bridge is:

$$
\operatorname{Fun}(T D, r, s)=\left\{\begin{array}{c}
\frac{T(r, s) \bullet[\eta(r, s)]^{\beta}}{\sum T(r, s) \bullet[\eta(r, s)]^{\beta}} \rightarrow \text { if ... route... found } \\
0 \rightarrow \text { otherwise }
\end{array}\right\}
$$

Where $\mathrm{T}(\mathrm{r}, \mathrm{s})$ is the pheromone deposited in the path between ' $r$ ' and ' $s$ ', $n(r, s)$ is the corresponding heuristic value which is the inverse of length of the particular path. $\beta$ is a parameter which determines the relative importance of pheromone versus distance $(\beta>$ $0)$. The pheromone update policy is as follows:

$$
T(r, s) \leftarrow(1-\alpha) \bullet T(r, s)+\sum(1-\alpha) \bullet T(r, s)--(4)
$$

Where, $\mathrm{L}$ is the length of tour, $\alpha$ is the pheromone decay parameter which is lies between ' 0 to 1 '. The pheromone values of each entry in the table can be initialized to equal values, thus providing nonbiased search for the best path. If some information about the best path is available, the pheromone values of the entry can be set to closer values to the optimum, thus, speed up the algorithm.

As in most table routing algorithms, the proposed routing protocol also provides the source with multiple paths to the destination. These paths are defined its priority and ready to be used in case of the failure of the best path in case of single path routing algorithm and al the paths are used for communication and number of paths may be desired as shown in the above formula. When a node wants to find and/or maintain a path to a destination, it sends forward ants (FA) searching for this destination. Both intermediate and source nodes forward the FA in the same way. A FA carries the path source address, the destination address, the inter-mediate node's ids, and the path information. The FA generation rate can be a function of network dynamics, data rate, time, etc. A FA moves in the network searching for the destination using the intermediate nodes' probability routing tables. The selection of the next neighbor is done randomly according to the probability distribution function.

A sufficient number of the ants will visit the neighbor corresponding to the highest probability in the routing table, however a number of the FA still have a probability to visit other nodes and other paths still have a probability to be visited. This will increase the number of the FA visiting nodes in the region around the best path. In addition, it allows a fair number of FA to visit other regions in the network. Unlike flooding, in this paper, a FA will be forwarded to only one neighbor. When a FA reaches its destination, the information carried by this FA path will be graded. Then, the FA will be killed and a backward ant will be generated. A backward ant carries its corresponding FA's path grade and path's intermediate nodes ids ant it will be send back following the reverse path of its corresponding FA. As the backward ant moves in the reverse path, the intermediate nodes modify their pheromone table based on the path grade carried by the backward ant and accordingly update their probability routing tables. Finally, the source node receives the backward ant, updates its tables, and kills the backward ant. The detailed proposed algorithm is given below:

\subsection{The Remodeled Ant Colony Optimization Algorithm:}

1) //Initialization Phase

For each pair $(r, s)$, the value of $\tau(r, s):=\tau_{0}$ Endfor

For $k:=1$ to $m$ do

Let $\left(r, k_{l}\right)$ be the starting city for an ant $k$

$$
J_{k}\left(r_{k l}\right):=\{1, \ldots, n\}-r_{k l}
$$




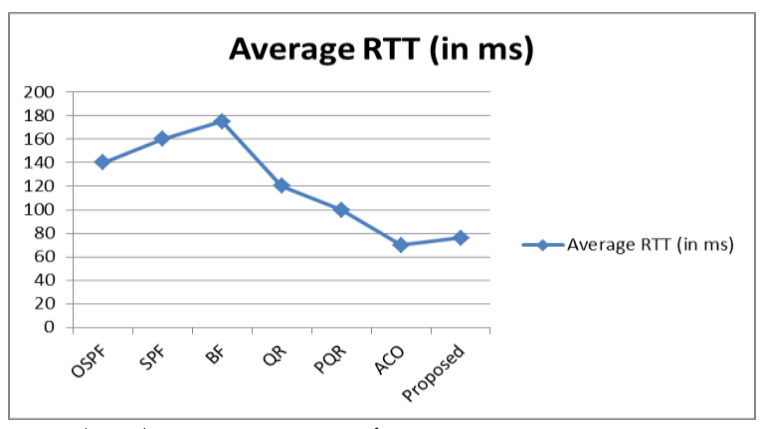

a) Average response time

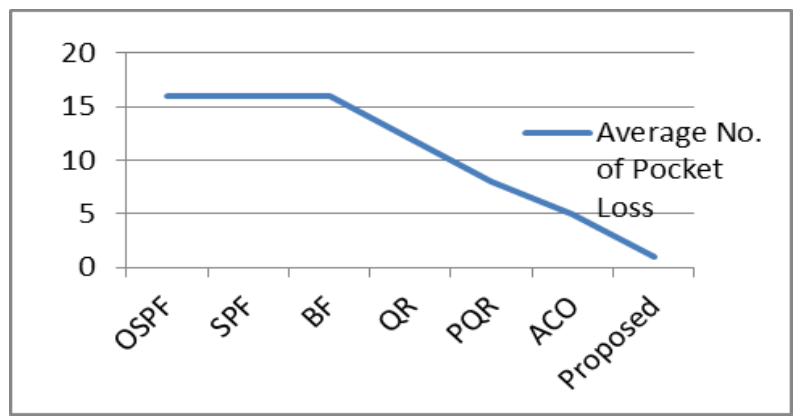

C) Average Packet Loss

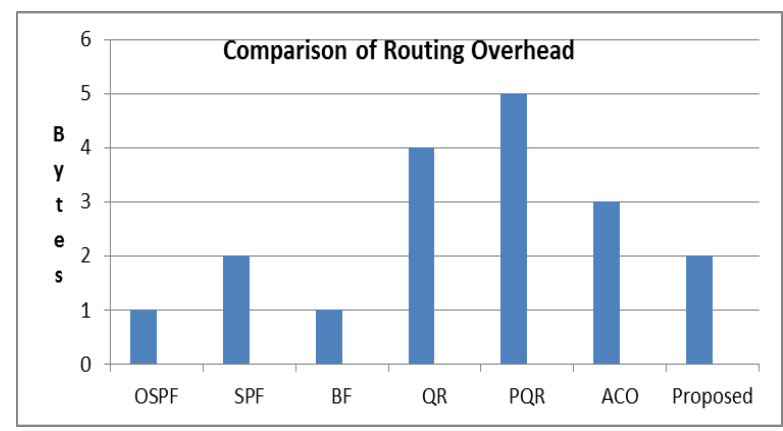

b) Routing overhead

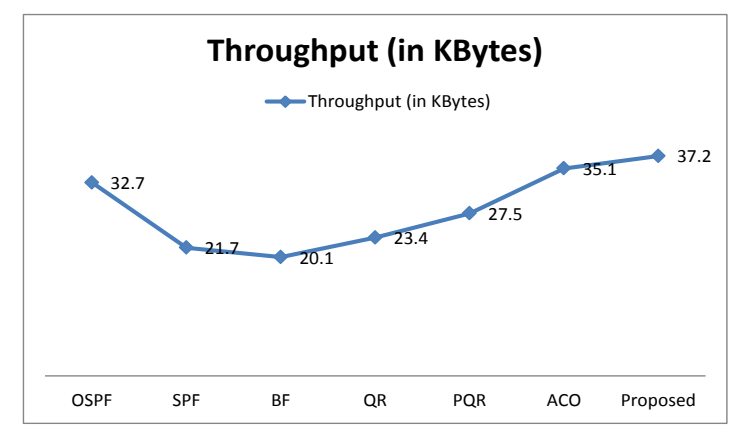

d) Throughput

Fig 4 Performance comparison between various routing algorithms with Proposed System (10 wired, 20Wireless and 5Mesh Nodes in heterogeneous network environment)

$/ / J_{k}\left(r_{k 1}\right)$ is the set of yet to be visited cities for //ant $k$ in city $r_{k l}$

$r_{k}:=r_{k 1}$

$/ / r_{k}$ is the city where ant $k$ is located

End-for

2) //This is the phase in which ants build their tours. The tour of ant $k$ is stored in //Tour ${ }_{\mathrm{k}}$

For $i:=1$ to $n$ do

If $i<n$

Then

For $k:=1$ to $m$ do

Choose the next city $S_{\mathrm{k}}$

$J_{k}\left(S_{k}\right):=J_{k}\left(r_{k}\right)-S_{k}$

$\operatorname{Tour}_{k}(i):=\left(r_{k}, S_{k}\right)$

End-for

Else

For $k:=1$ to $m$ do

//In this cycle all the ants go back

to the initial city $r_{k l}$

$S_{k}:=r_{k 1}$

End-for

$\operatorname{Tour}_{k}(i):=\left(r_{k}, S_{k}\right)$

End-if
//In this phase local updating occurs and pheromone is updated

For $k:=1$ to $m$ do

$$
\begin{aligned}
& T(r, s) \leftarrow(1-\alpha) \bullet T(r, s)+\sum(1-\alpha) \bullet T(r, s) \\
& r_{k}:=s_{k} / / \text { New city for ant } k
\end{aligned}
$$

End-for

End-for

3) //In this phase global updating occurs and pheromone is updated

For $k:=1$ to $m$ do

Compute $L_{k} / / L_{k}$ is the length of the tour done by ant $k$

End-for

Compute $L_{\text {best }}$

/*Update edges belonging to $L_{\text {best }}$

For each edge $(r, s)$

$T(r, s) \leftarrow(1-\alpha) \bullet T(r, s)+\sum(1-\alpha) \bullet T(r, s)$

End-for

4) $/ /$ In this phase assigns priority

For $k:=1$ to $m$ do

Sort the routing table based on pheromone values 
Table 1: Routing Table of Node ' $\mathrm{S}$ ' in case of Single path routing algorithm

\begin{tabular}{|l|l|l|l|l|l|}
\hline Dest. & Next Hop & Priority & $\begin{array}{l}\text { Pheromone } \\
\text { Density }\end{array}$ & Availability & Status \\
\hline $\mathrm{D}$ & $\mathrm{I}_{1}$ & 1 & 20 & Available & Route Selected \\
\hline $\mathrm{D}$ & $\mathrm{I}_{2}$ & 2 & 15 & Available & Route in wait list \\
\hline $\mathrm{D}$ & $\mathrm{I}_{3}$ & $\infty$ & 15 & Not Available & Routes Removed \\
\hline
\end{tabular}

Table 2: Routing Table of Node ' $\mathrm{S}$ ' in case of Multi path routing algorithm

\begin{tabular}{|l|l|l|l|l|l|l|}
\hline Dest. & $\begin{array}{l}\text { Next } \\
\text { Hop }\end{array}$ & Priority & $\begin{array}{l}\text { Pheromone } \\
\text { Density }\end{array}$ & $\begin{array}{l}\text { Probability } \\
\text { (Pheromone) }\end{array}$ & Availability & Status \\
\hline $\mathrm{D}$ & $\mathrm{I}_{1}$ & 1 & 20 & 0.7 & Available & $70 \%$ packets transmitted \\
\hline $\mathrm{D}$ & $\mathrm{I}_{2}$ & 2 & 15 & 0.3 & Available & $30 \%$ packets transmitted \\
\hline $\mathrm{D}$ & $\mathrm{I}_{4}$ & $\infty$ & 15 & -- & Not Available & Routes Removed \\
\hline
\end{tabular}

Assigns high priority to higher pheromone density path

Choose the best path based on priority in the wired network and availability $\&$ priority in the case of wireless network

\}

End-for

This proposed routing is simulated in NS2. The result and performance analysis is described in the coming section.

\section{Result and Analysis:}

The Proposed algorithm follows the priority as additional decision factors in the case of single path and multipath routing. The sample routing table and the communication status for single path and multipath routing protocols are shown in Table 1 and Table 2 respectively. If the Response Complexity of particular node is negative then which priority is assigned as "infinity". Suppose there are no such paths having positive response complexity then the lowest RTT path is shown as best path in the case of single path.

\section{Conclusion}

ACO is implemented in always all engineering applications like continuous casting of steel [38], data reconciliation and parameter estimation in dynamic systems [39], gaming theory [40], In-Core Fuel Management Optimization in Nuclear Engineering [41], target tracking problem in signal processing [42], design of automatic material handling devices [43], Mathematical and kinetic modeling of bio-film reactor [44], optimization of a rail vehicle floor sandwich panel [45], software design [46], Vehicle routing design [47], Quadratic Assignation problem [48], mutation problem [49]. The experimental of ACO shows [50] [51] that the ACO outperforms than the existing research methodologies. A minute redefinition, updation and or modification of the procedural steps of ACO also will raise the performance dramatically.

\section{References :}

1. P.-P. Grasse, "La reconstruction du nid et les coordinations interindividuelles chez Belli-cositermes Natalensis et Cubitermes sp. Lath' eorie de la stigmergie: Essai d'interpr' etation du comportement des termites constructeurs," Insectes Sociaux, vol. 6, pp. 41-81, 1959.

2. J.-L. Deneubourg, S. Aron, S. Goss, and J.-M. Pasteels, "The self-organizing exploratory pattern of the Argentine ant," Journal of Insect Behavior, vol. 3, p. 159, 1990.

3. S. Goss, S. Aron, J.-L. Deneubourg, and J.-M. Pasteels, "Self-organized shortcuts in the Argentine ant," Naturwissenschaften, vol. 76, pp. 579-581, 1989.

4. J.-M. Pasteels, J.-L. Deneubourg, and S. Goss, "Selforganization mechanisms in ant societies (i): Trail recruitment to newly discovered food sources," Experientia Supplementum, vol.54, p. 155, 1987.

5. M. Dorigo, V. Maniezzo, and A. Colorni, "Positive feedback as a search strategy," Dipartimento di Elettronica, Politecnico di Milano, Italy, Tech. Rep. 91016, 1991. 
6. M. Dorigo, "Optimization, learning and natural algorithms (in italian)," Ph.D. dissertation, Dipartimento di Elettronica, Politecnico di Milano, Italy, 1992.

7. M. Dorigo, V. Maniezzo, and A. Colorni, "Ant System: Optimization by a colony of cooperating agents," IEEE Transactions on Systems, Man, and Cybernetics-Part B, vol. 26, no. 1, pp. 29-41, 1996.

8. M. Dorigo, T. Stutzle, "Ant Colony Optimization”, MIT Press, Cambrige, 2004

9. L.M. Gambardella and M. Dorigo, "Ant-Q: A reinforcement learning approach to the traveling salesman problem," in Proc. Twelfth International Conference on Machine Learning (ML-95), A. Prieditis and S. Russell, Eds., Morgan Kaufmann Publishers, pp. 252-260, 1995.

10. M. Dorigo and L.M. Gambardella, "Ant colonies for the traveling salesman problem," BioSystems, vol. 43, no. 2, pp. 73-81, 1997.

11. T. St " utzle and H.H. Hoos, "Improving the Ant System: A detailed report on the MAX-MIN Ant System," FG Intellektik, FB Informatik, TU Darmstadt, Germany, Tech. Rep. AIDA-96-12, Aug. 1996.

12. B. Bullnheimer, R.F. Hartl, and C. Strauss, "A new rank based version of the Ant System-a computational study," Institute of Management Science, University of Vienna, Tech. Rep., 1997.

13. V. Maniezzo, "Exact and approximate nondeterministic tree-search procedures for the quadratic assignment problem," INFORMS Journal on Computing, vol. 11, no. 4, pp. 358-369, 1999.

14. O. Cordon, I.F. de Viana, F. Herrera, and L. Moreno, "A new ACO model integrating evolutionary computation concepts: The best-worst Ant System," in Proc. ANTS 2000, M.Dorigo et al., Eds., IRIDIA, Universit'e Libre de Bruxelles, Belgium, pp. 22-29, 2000.

15. C. Blum, A. Roli, and M. Dorigo, "HC-ACO: The hypercube framework for Ant Colony Optimization," in Proc. MIC'2001-Metaheuristics International Conference, vol. 2, Porto, Portugal, pp. 399-403, 2001.

16. Li-Ning Xing, Ying-Wu Chen, PengWang, Qing-Song Zhao, Jian Xiong, "A Knowledge-Based Ant Colony Optimization for Flexible Job Shop Scheduling Problems", Applied Soft Computing 10 (2010) 888-896

17. Wang Chen, Yan-jun Shi, Hong-fei Teng, Xiao-ping Lan, Li-chen $\mathrm{Hu}$, "An efficient hybrid algorithm for resourceconstrained project scheduling", Information Sciences 180 (2010) 1031-1039
18. C. Twomey, T. Stützle, M. Dorigo, M. Manfrin, M. Birattari, "An analysis of communication policies for homogeneous multi-colony ACO algorithms", Information Sciences 180 (2010) 2390-2404

19. Komarudin, Kuan Yew Wong, "Applying Ant System for solving Unequal Area Facility Layout Problems", European Journal of Operational Research 202 (2010) 730-746

20. A. Berrichi, F. Yalaoui, L. Amodeo, M. Mezghiche, "Computers Bi-Objective Ant Colony Optimization approach to optimize production and maintenance scheduling”, Operations Research 37 (2010) 1584-1596

21. Hsin-Yun Lee, Hao-Hsi Tseng, Meng-Cong Zheng, PeiYing Li, "Decision support for the maintenance management of green areas", Expert Systems with Applications 37 (2010) 4479-4487

22. Wei-Neng Chen, Jun Zhang, Henry Shu-Hung Chung, Rui-Zhang Huang, and Ou Liu, "Optimizing Discounted Cash Flows in Project Scheduling-An Ant Colony Optimization Approach", IEEE TRANSACTIONS ON SYSTEMS, MAN, AND CYBERNETICS-PART C: APPLICATIONS AND REVIEWS, VOL. 40, NO. 1, JANUARY 2010

23. W. Herroelen, B. D. Reyck, and E. Demeulemeester, "Resource constrained project scheduling, a survey of recent developments," Comput. Oper. Res., vol. 13, no. 4, pp. 279-302, 1998

24. P. Brucker, A. Drexl, R.Mohring, K. Neumann, and E. Pesch, "Resource constrained project scheduling: Notation, classification,models and methods," Eur. J. Oper. Res., vol. 112, pp. 3-41, 1999

25. L. Ozdamar and G. Ulusoy, "A survey on the resourceconstrained project scheduling problem," IIE Trans., vol. 27, pp. 574-586, 1995

26. G.Ulusoy, "Four payment models for the multi-mode resource constrained project scheduling problem with discounted cash flows," Ann. Oper. Res., vol. 102, pp. 237-261, 2001

27. I.T. Tanev, T. Uozumi, Y.Morotome, Hybrid evolutionary algorithm-based real-world Flexible Job Shop Scheduling Problem: application service provider approach, Applied Soft Computing 5 (1) (2004) 87-100.

28. Manuel Lopez-Ibanez, Christian Blum, "Beam-ACO for the travelling salesman problem with time windows", Computers \& Operations Research 37 (2010) 1570-1583

29. Frank Neumanna, CarstenWitt, "Ant Colony Optimization and the minimum spanning tree problem", Theoretical Computer Science 411 (2010) 2406-2413 
30. Xiao-ming You, Sheng Liu, Yu-ming Wang, "Quantum Dynamic Mechanism-based Parallel Ant Colony Optimization Algorithm", International Journal of Computational Intelligence Systems, Suppl. 1 (December, 2010), 101-113

31. Ali Maroosi, Babak Amiri, "A new clustering algorithm based on hybrid global optimization based on a dynamical systems approach algorithm", Expert Systems with Applications 37 (2010) 5645-5652

32. Jiejin Cai, Xiaoqian Ma, Qiong Li, Lixiang Li, Haipeng Peng, "A multi-objective chaotic ant swarm optimization for environmental/economic dispatch", Electrical Power and Energy Systems 32 (2010) 337-344

33. Saravuth Pothiya, Issarachai Ngamroo, Waree Kongprawechnon, "Ant colony optimisation for economic dispatch problem with non-smooth cost functions", Electrical Power and Energy Systems 32 (2010) 478-487

34. Abbas Ketabi, Ahmad Alibabaee, R. Feuillet, "Application of the ant colony search algorithm to reactive power pricing in an open electricity market", Electrical Power and Energy Systems 32 (2010) 622-628

35. Taher Niknam, Hamed Zeinoddini Meymand, Majid Nayeripour, "A practical algorithm for optimal operation management of distribution network including fuel cell power plants", Renewable Energy 35 (2010) 1696-1714

36. Sudip Misra, Sanjay K. Dhurandher, Mohammad S. Obaidat, Karan Verma, Pushkar Gupta, “A low-overhead fault-tolerant routing algorithm for mobile ad hoc networks: A scheme and its simulation analysis", Simulation Modelling Practice and Theory 18 (2010) 637-649

37. Zhiding Yu, Oscar $\mathrm{C} \mathrm{Au}$, Ruobing Zou, Weiyu Yu, Jing Tian, "An adaptive unsupervised approach toward pixel clustering and color image segmentation", Pattern Recognition 43 (2010) 1889-1906

38. C.A. Santosa, J.A. Spimb, A. Garcia, "Mathematical modeling and optimization strategies (genetic algorithm and knowledge base) applied to the continuous casting of steel”, Engineering Applications of Artificial Intelligence 16 (5-6) (2003) 511-527

39. P. Vachhani, R. Rengaswamy, V. Venkatasubramanian, "A framework for integrating diagnostic knowledge with nonlinear optimization for data reconciliation and parameter estimation in dynamic systems", Chemical Engineering Science 56 (6) (2001) 2133-2148

40. Jose A. Mocholi, Javier Jaen, Alejandro Catala, Elena Navarro, “An emotionally biased ant colony algorithm for pathfinding in games", Expert Systems with Applications 37 (2010) 4921-4927

41. Anderson Alvarenga de, Moura Meneses, Luca Maria Gambardella, Roberto Schirru, "A new approach for heuristics-guided search in the In-Core Fuel Management Optimization", Progress in Nuclear Energy 52 (2010) 339-351

42. Benlian Xu, Qinglan Chen, Jihong Zhu, Zhiquan Wang, "Ant estimator with application to target tracking", Signal Processing 90 (2010) 1496-1509

43. Babak Shirazi, Hamed Fazlollahtabar, Iraj Mahdavi, "A six sigma based multi-objective optimization for machine grouping control in flexible cellular manufacturing systems with guide-path flexibility", Advances in Engineering Software 41 (2010) 865-873

44. K. Rama Rao, T. Srinivasan, Ch. Venkateswarlu, "Mathematical and kinetic modeling of biofilm reactor based on ant colony optimization", Process Biochemistry 45 (2010) 961-972

45. Craig W. Hudson, Joe J. Carruthers, A. Mark Robinson, "Multiple objective optimisation of composite sandwich structures for rail vehicle floor panels", Composite Structures 92 (2010) 2077-2082

46. A. Bouchachia, R. Mittermeir, P. Sielecky, S. Stafiej, M. Zieminski, "Nature-inspired techniques for conformance testing of object-oriented software", Applied Soft Computing 10 (2010) 730-745

47. L. Barcos, V. Rodríguez, M.J. Álvarez, F. Robusté, "Routing design for less-than-truckload motor carriers using Ant Colony Optimization", Transportation Research Part E 46 (2010) 367-383

48. Amilkar Puris, Rafael Bello, Francisco Herrera, "Analysis of the efficacy of a Two-Stage methodology for ant colony optimization: Case of study with TSP and QAP”, Expert Systems with Applications 37 (2010) 5443-5453

49. Nan Zhao, Zhilu Wu, Yaqin Zhao, Taifan Quan, "Ant colony optimization algorithm with mutation mechanism and its applications", Expert Systems with Applications 37 (2010) 4805-4810

50. B. Chandra Mohan, Prasanna, Anand, D. Sridharan, "AntNet - Ant based Routing Protocol with OSPF", IEEE International Conference on Emerging Trends in High Performance Architecture algorithms and Computing, 2007

51. B. Chandra Mohan, R. Baskaran, 'Improving network efficiency by optimal load balancing using redundant link 
avoidance algorithm', International Journal of Computer science Issues, Vol 3, Mar 2010.

52. D. Subramanian, P. Druschel, and J. Chen, "Ants and reinforcement learning: A case study in routing in dynamic networks," in Proc. Int. Joint Conf. Artificial Intelligence, Palo Alto, CA, 1997, IJCAI-97, pp. 832838.

53. M. Heusse, D. Snyers, S. Guérin, and P. Kuntz, "Adaptive Agent-Driven Routing and Load Balancing in Communication Networks," ENST Bretagne, Brest, France, Tech. Rep. RR-98 001-IASC, 1998

54. G. D. Caro and M. Dorigo, "AntNet: A Mobile Agents Approach to Adaptive Routing," Univ. Libre de Bruxelles, Brussels, Belgium, Tech. Rep. IRIDIA/97-12, 1997

\section{Authors:}

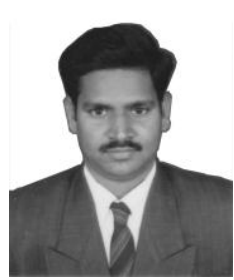

[*] Mr. Chandra Mohan B received his Diploma in Electrical and Electronics Engineering, Bachelor Degree in Information Technology and Master Degree in Computer Science and Engineering from State Board, Madras University and Anna University in the years 1993, 2003 and 2006 respectively. He worked both industry and academic in India and Abroad. Presently he is working as Principal in LCR College of Engineering \& Technology, Chennai, India. He was with the Anna University, Chennai, India and Kuwait National Petroleum Company, Kuwait. His present research included Networks, Data Mining and Swarm intelligence. He motivating more than 10000 active students in the NS2 based simulation environment. He has published 8 papers in National, International Conferences, International Journals, and Springer Book series. His paper awarded as best paper in IEEE SNPD 2008, Thailand. He is a reviewer in IEEE, ACM, Springer, Elsevier and dozens of international Journals. He has visited Kuwait. He is a life member of Indian Society for Technical Education (ISTE), Institution of Electronics and Telecommunication Engineers (IETE), Computer Society of India (CSI) and International Association for Engineers (IAEng).

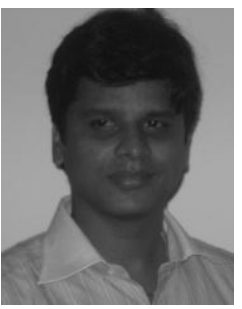

[**]Dr. Baskaran $\mathbf{R}$ received his Bachelor Degree in Electrical and Electronics Engineering, Master Degree in Computer Science and Engineering from Madras University and Doctorate from Anna University in the years 2000, 2001, and 2007 respectively. He is now working as an Assistant Professor in Department of Computer Science and Engineering, Anna University Chennai. His present research includes Networks, Database and Image Processing. He has published 35+ papers in International, National Journals and Conferences. He is a reviewer in IEEE, ACM, Springer, Elsevier and dozens of international Journals. He is a life member of Institution of Electronics and Telecommunication Engineers (IETE), Indian Society for Technical Education (ISTE), Computer Society of India (CSI) and International Association for Engineers (IAEng). 